\title{
An Investigation of Initial Shock Cell Formation in Turbulent Coanda Wall Jets
}

\author{
Caroline P. Lubert \\ James Madison University, Virginia, USA
}

Christian R. Schwantes

Stanford University, California, USA

\author{
Richard J. Shafer \\ University of Colorado Boulder, Colorado, USA
}

(Received 1 July 2014; accepted 30 September 2015)

Turbulent Coanda wall jets are present in a multitude of applications. ${ }^{1}$ Their obvious advantages for flow deflection are often outweighed by disadvantages related to the increased noise levels associated with such jets. Better predictions of Coanda jet noise would allow the Coanda effect to be more widely applied, and its potential to be fully realized. This paper applies the method of characteristics to a steady two-dimensional axisymmetric supersonic flow in order to determine the location of the first shock cell downstream of the nozzle. This phenomenon has previously been found to be particularly important in determining both the OASPL and peak frequency of the broadband high-frequency Shock-Associated Noise (BBSAN) emitted by a given jet configuration. ${ }^{10,20}$ The current work has also illuminated the relationship between cell location and flow characteristics, and thus the effect of jet operating conditions on BBSAN can now be determined. ${ }^{11}$ The relationship between cell location and jet breakaway is also under investigation. Predictions are compared with experimental results obtained using flow visualization techniques. This work is in the process of being extended so that the Rankine-Hugoniot conditions can be used to predict the shock cell structure (and thus the BBSAN) along the entire jet. ${ }^{22}$

\section{NOMENCLATURE}

$C_{+} \quad$ Characteristic moving towards Coanda surface

$C_{-} \quad$ Characteristic moving away from Coanda surface

a Speed of sound $(\mathrm{m} / \mathrm{s})$

$(x, y) \quad$ Cartesian coordinates of point of interest

$(u, v) \quad$ Velocity components at point of interest $(x, y)$ along the $x$ and $y$-axes respectively $(\mathrm{m} / \mathrm{s})$

$\theta \quad$ Angle streamline makes with $x$-axis

$\alpha \quad$ Mach angle

$\lambda_{+} \quad$ Slope of characteristic moving towards Coanda surface

$\lambda_{-} \quad$ Slope of characteristic moving away from Coanda surface

$\delta \quad$ Dirac delta function. $\delta=1$ : axisymmetric flow, $\delta=0$ : planar 2D flow

$\gamma \quad$ Ratio of specific heat capacities

$R_{c} \quad$ Radius of circular part of flare (m)

$R_{f} \quad$ Radius of interior stem of flare (m)

$h \quad$ Exit slot (mm)

$y_{0} \quad y$-value assigned at lip (m)

$p_{e} \quad$ Nozzle exit pressure (psig)

$p_{a} \quad$ Ambient (atmospheric) pressure (psig)

$p_{o} \quad$ Reservoir pressure (psig)

$M_{e} \quad$ Exit Mach number

$U_{j x} \quad$ Jet exit velocity $(\mathrm{m} / \mathrm{s})$

\section{INTRODUCTION}

\subsection{The Coanda Effect}

The Coanda effect, discovered early in the twentieth century by Romanian mathematician and scientist Henri Coanda, is the phenomenon whereby '.. when a jet is passed over a curved surface it bends to follow the surface, entraining large amounts of air as it does so...' ${ }^{1-3}$ Consider a fluid element exiting a nozzle adjacent to a curved surface. The radial equilibrium of the element leads to the development of a pressure field which forces the fluid against the surface, and this effect is reinforced by the slightly enhanced viscous drag which is experienced by the jet on its wall side as it exits the nozzle, and which also tends to deflect it towards the wall. Subsequently, this pressure field will continue to force the jet towards the surface. An additional viscous effect, namely the entrainment of the ambient fluid between the jet and the surface, may also help to move the jet towards the wall. The effect breaks down under certain operating conditions, at which point jet breakaway occurs. A hysteresis effect is subsequently observed. The Coanda effect is noticed in the natural world (with both positive and negative consequences) and is frequently invoked in aeronautics, maritime technology and industrial engineering. ${ }^{1}$ The substantial flow deflection offered by the Coanda principle is generally accompanied by enhanced levels of turbulence and increased entrainment. A direct consequence of these effects is often a significant escalation in the associated noise levels, and it is posited that this disadvantage has prevented its application from becoming more widespread in recent years. ${ }^{4}$ Clearly, bet- 
ter understanding of the noise emission characteristics of turbulent Coanda jets will facilitate improvements in prediction and attenuation of such noise.

\subsection{Principal Noise Sources Associated with Turbulent Coanda Wall Jets}

The jet under consideration here is assumed to issue at high velocity (200-500 m/s) from an annular exit slot. Immediately upon exit it is adjacent to a solid three-dimensional Coanda surface. More detailed information concerning the geometry of interest is shown in Fig. 1. Although this representation is that of a Coanda flare of the type used in the petroleum indus$\operatorname{try}^{5,6}$ (an example of which is shown in operation in Fig. 2), the experimental methods and models developed herein can easily be applied to other types of three-dimensional Coanda jet flows.

Although Coanda jets emit both low- and high-frequency noise, it is the latter that is generally the most destructive, irritating and also the easiest to attenuate. Both broadband noise and discrete tones are emitted, although the appearance of the tones is rather sporadic. Thus, the focus of the current work is on the broadband high-frequency noise. There are two principal sources of such noise in Coanda jets; turbulent mixing noise (TMN) and shock-associated noise (SAN). A comprehensive investigation of TMN associated with these jets has already been undertaken. ${ }^{7-9}$ The other principal high frequency acoustic source commonly observed in such flows is SAN, and this paper presents the results of a preliminary investigation into the SAN generated by the mixing layer of a threedimensional turbulent jet flowing adjacent to a solid Coanda surface.

As mentioned previously, configurations such as that shown in Fig. 1 emit both low- and high-frequency noise. It is the latter that is of greatest interest, since it is both the most annoying to the human ear, and the easiest to attenuate. This paper will focus on SAN.

\subsection{Shock Associated Noise (SAN)}

It is well known that, in contrast to subsonic jets, conditions at a downstream point in a supersonic jet cannot affect those upstream. ${ }^{10}$ In this way, discontinuities in flow properties can arise. Depending upon the relative pressure difference between the nozzle exit pressure $\left(p_{e}\right)$ and the ambient pressure $\left(p_{a}\right)$ a shock cell structure is formed in the jet plume close to the jet exit slot. The interaction between this structure and the large-scale coherent turbulence in the jet shear layer generates the high-frequency sound known as SAN. This phenomenon has been studied in great detail for free jets. ${ }^{11-15}$ It has previously been noted that turbulent Coanda wall jets display at least some similar characteristics to their two-dimensional counterparts, and thus in order to facilitate a preliminary investigation into the nature and behavior of SAN in Coanda flows, this simplifying assumption will be made. ${ }^{7}$ However, when the flow is three-dimensional, complicating factors such as radial expansion and streamline curvature are present, and should be accounted for in future models.

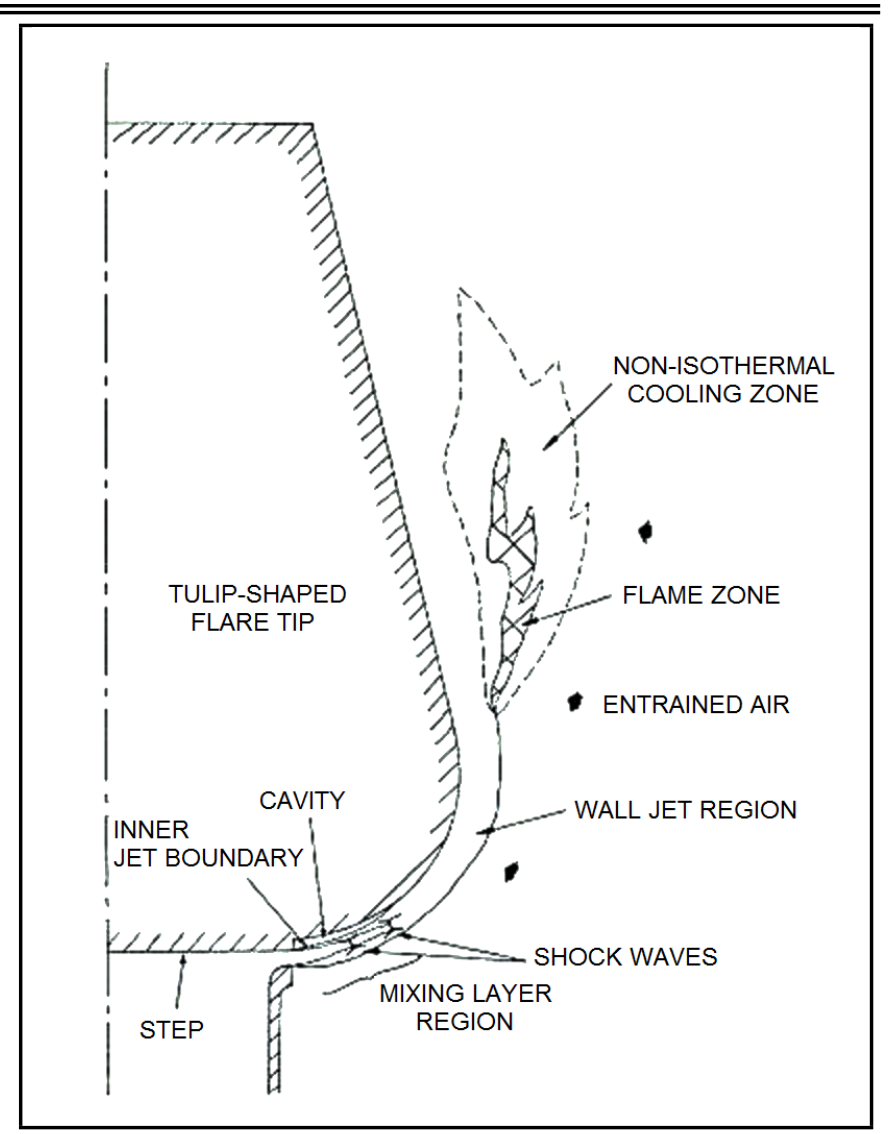

Figure 1. The flow field and combustion zone of a Coanda flare.

\section{SHOCK WAVES IN COANDA FLOWS}

The flow under consideration in the current work is that associated with a turbulent Coanda flare of the type shown in Fig. 1. Green has shown that one-dimensional flow theory can be used to describe the flow through a convergent-divergent nozzle of the kind present in the Coanda flare. ${ }^{10}$ Thus, the jet emerging from the exit slot is supersonic for almost all operating pressures, and shock waves are formed in the vicinity of the nozzle exit. The exact location of these shock waves depends upon the relative magnitudes of the pressure in the reservoir supplying the nozzle, $p_{0}$, and the pressure of the medium into which the jet flows, known as the ambient or back pressure, $p_{a}$. Assuming that flow in the divergence is isentropic, the jet pressure at exit, $p_{e}$, is given by

$$
\left.p_{e}=p_{0}\left[1+\left(\frac{\gamma-1}{2}\right) M_{e}^{2}\right)\right]^{\frac{-\gamma}{\gamma-1}}
$$

where $p_{0}$ is the pressure in the reservoir supplying the nozzle, $\gamma$ is the ratio of specific heats and $M_{e}$ is the exit Mach number. ${ }^{10}$

If the pressure at the nozzle exit, $p_{e}$, equals the ambient pressure, i.e. $p_{e}=p_{a}$, then the jet is said to be correctly expanded. In this case, the jet is parallel-sided with a uniform Mach number throughout, and is free of shock waves. In general, Coanda flare jets are rarely perfectly-expanded in practice, but are typically either under- or over-expanded, and an adjustment of the exit pressure via compression (condensation) or expansion (rarefaction) waves will occur. This leads to the formation of a shock cell structure within the mixing layer region of the jet. See Fig. 1.

Consider, for example, an under-expanded jet. In this case, $p_{e}>p_{a}$ and a fan of expansion waves (waves such that the 


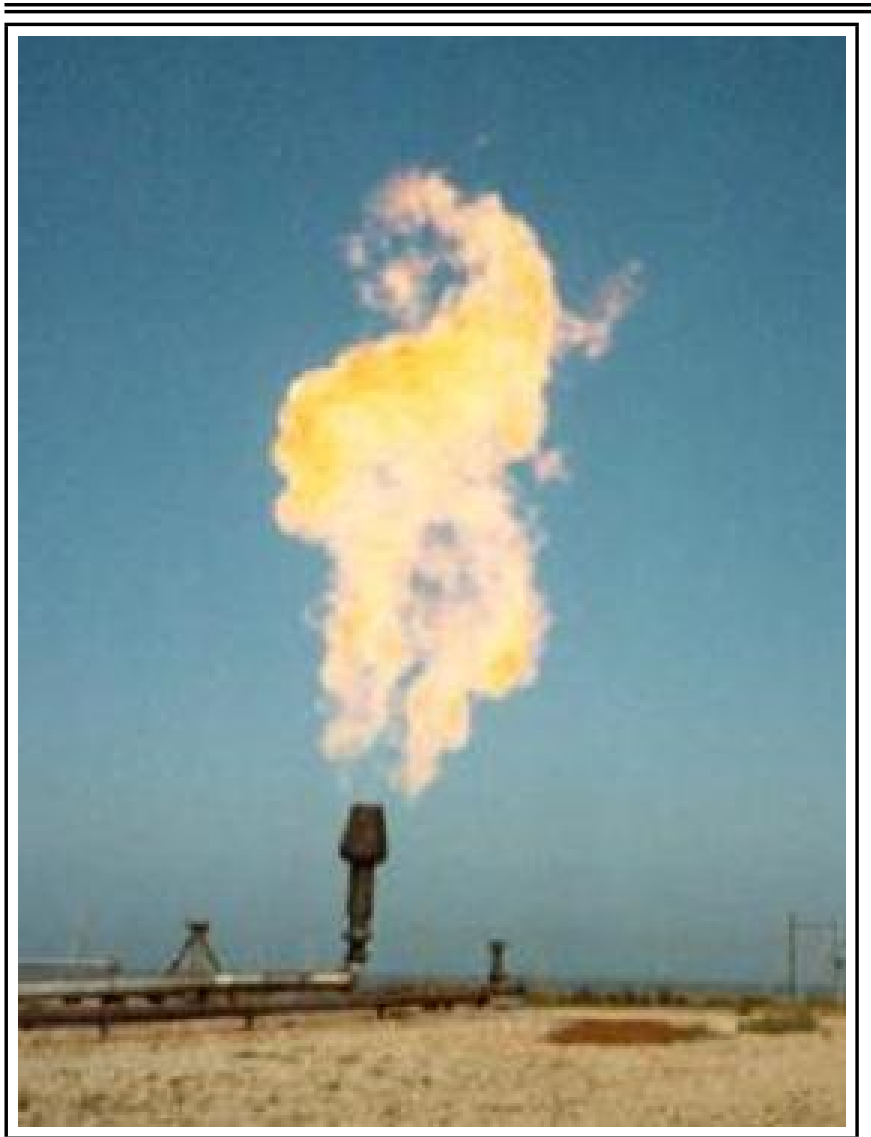

Figure 2. An operating Coanda flare.

pressure and density of a base flow decrease on crossing them) will be generated at the lip of the nozzle exit slot. On interacting with a free jet boundary, the expansion waves cause the boundary to be displaced outwards, and this effect can be seen in Fig. 3. The expansion waves will reflect as either expansion or compression waves as they interact with a boundary. In order to preserve constant pressure at the jet boundary, the incident and reflected waves will be of opposite kinds and so an expansion wave reflects as a compression wave (pressure and density increase on crossing it) and vice versa. However, when a wave reflects from a solid surface such as the flare-tip, zero normal velocity must be preserved at the wall, and so an expansion wavefront will be reflected as an expansion wavefront, for example.

The coalescence of several compression waves forms a shock wave, which reflects as a shock wave from the solid flare tip surface and then reflects at the jet boundary as an expansion wave. The pattern of expansion waves, compression waves, and shock waves repeats itself periodically and the quasi-periodic constituents of this pattern, known as shock cells, are shown schematically in Fig. 1. Typically a series of 6-10 shock cells will form in the jet exhaust. Figure 3 shows the flow structure typically observed in the mixing layer region of the jet. Shock cells are clearly seen as light and dark lines in the figure. Turbulent eddies convected downstream within the mixing layer region of the jet cause these shocks to be deformed. This distortion of the shock front propagates away as the broadband, but strongly peaked sound waves known as SAN. This acoustic phenomenon has several interesting aspects. Firstly, there is a strong directivity associated with the SAN emitted by moving sound waves. It has been observed

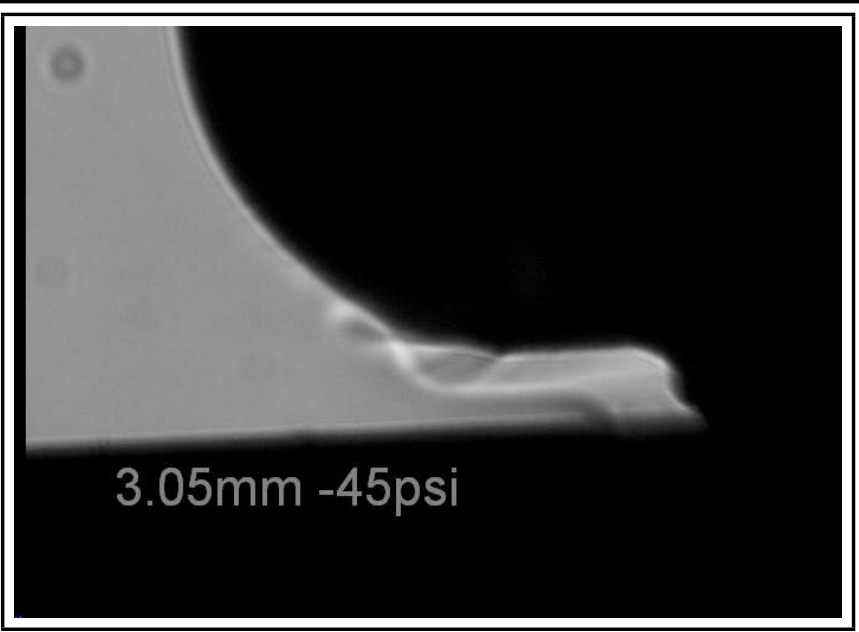

Figure 3. Typical flow structure in Coanda flare mixing layer. Slot width, $h=3.05 \mathrm{~mm}$, operating pressure $=45 \mathrm{psig}$.

that a turbulent eddy can successively interact with several shock waves, generating multiple sound sources (one resulting from each interaction). ${ }^{11}$ Additionally, a feedback cycle is often present, leading to the generation of discrete, harmonically related tones known as screech tones.

The Coanda flare being studied is convergent-divergent and supersonic under most operating conditions. It is well known that the flow just downstream of the jet exit slot is responsible for most of the flare noise generation and that most of the SAN is produced in this region. ${ }^{10}$ Thus in order to fully comprehend this high-frequency acoustic emission, it is very important to understand the behaviour of the flow in the initial region near the nozzle exit. Green has previously shown that one-dimensional flow theory and the method of characteristics can be used to describe flow through this nozzle, at least until the first shock is formed. ${ }^{7,10,17,18}$ The method of characteristics has previously been applied ${ }^{19,20}$ to the inviscid core of a supersonic jet (following the method of Dash et al. ${ }^{21}$ whereby the outer shear layer and surface boundary layer are ignored). In this case, the aim of modelling the jet structure (particularly, first shock cell location) was so that jet breakaway could be better predicted. ${ }^{19,20}$ Indeed, comparison of predictions with experimental results for the location of the first shock cell (at low blowing pressures and before any shock waves occur) is good. Specifically, according to Gilchrist and Gregory-Smith, 'The method of characteristics is shown to be adequate for calculating the [shock cell structure in the] inviscid core of the underexpanded [flat] jet'. ${ }^{20}$ In the current work, it is the location of the first shock cell that is of interest, since 'for high speed jets, the shock cell structure close to the slot is very important' ${ }^{20}$ Note that in a curved jet, the rapid growth of the outer shear layer (caused by streamline curvature) means that the shock cell structure is shorter than in equivalent plane jets. $^{20}$

\section{MATHEMATICAL DETERMINATION OF INITIAL SHOCK CELL LOCATION}

\subsection{Theory: Method of Characteristics}

For a steady two-dimensional irrotational flow, the governing equations are the speed of sound relationship, an equation expressing irrotationality and the Gas Dynamic equation. 
These form a system of two coupled quasi-linear nonhomogeneous partial differential equations (PDEs) of the first-order in two independent variables, $x$ and $y$. (Note that in this context, 'quasi-linear ... of the first-order' means that a PDE is nonlinear in the dependent variables ( $u$ and $v$, the flow velocity components) but linear in the first partial derivatives, $\left(u_{x}, u_{y}, v_{x}, v_{y}\right)$ of these dependent variables). These equations govern both subsonic and supersonic flow. However, the coefficients of the various derivatives are such that the mathematical type of the PDEs changes from elliptic when $M<1$ (i.e. subsonic flow) to hyperbolic for supersonic flow $(M>1)$. For the two-dimensional supersonic turbulent Coanda flow under consideration, these hyperbolic PDEs can be solved using the Method of Characteristics (MOC). Such equations have the property that they can be reduced to ordinary differential equations (ODEs) known as compatibility equations, which are valid along specific, related curves known as characteristics. Physically, characteristics represent the path of propagation of a physical disturbance.

For a steady two-dimensional irrotational flow, it is well known $^{16}$ that the governing equations yield compatibility equations

$$
\begin{aligned}
\left(u_{ \pm}^{2}-a_{ \pm}^{2}\right) d u_{ \pm}+ & {\left[2 u_{ \pm} v_{ \pm}-\left(u_{ \pm}^{2}-a_{ \pm}^{2}\right) \lambda_{ \pm}\right] d v_{ \pm}-} \\
& \frac{a_{ \pm}^{2} v_{ \pm}}{y_{ \pm}} d x_{ \pm}=0
\end{aligned}
$$

which are valid along the $C_{+}$(where + denotes the direction towards the flare tip surface) and $C_{-}$(away from the flare tip) characteristics described by

$$
\frac{d y}{d x_{ \pm}}=\lambda_{ \pm}=\tan \left(\theta_{ \pm} \mp \alpha_{ \pm}\right) ;
$$

where $\theta_{ \pm}$is the angle that the flow streamline makes with the $\mathrm{X}$-axis, and $\alpha_{ \pm}$is the Mach angle. Note the unusual sign convention used in Eq. (5). This follows that used by Green in which the $x$-axis is vertical and the $y$-axis is horizontal. ${ }^{10} \mathrm{In}$ the case of a supersonic flow, the characteristics are the Mach lines of the flow. Since Eqs. (2) and (3) are non-linear, they must be discretized and solved by numerical means. In the present work, following Green, the Euler predictor-corrector method is used. ${ }^{10}$

\subsection{Numerical Solution: Euler Predictor- Corrector Method}

Numerical determination of the location of the shock cells in a Coanda flare jet is based on a modified Euler predictorcorrector finite difference method. This is an iterative algorithm that proceeds in two steps. The prediction step calculates a rough approximation of the desired quantities and the corrector step then refines this initial approximation. At each point $(x, y)$ along the characteristics, the velocity components $(u, v)$ associated with that location $(x, y)$ must be determined. In order to do so, the region of interest must be divided into three separate areas: interior points (which have both $C_{+}$and $C_{-}$ characteristics), wall points (which have only $C_{+}$characteristics) and jet boundary points (with only $C_{-}$characteristics).

\subsubsection{Interior Points}

Equation (3) defines two characteristics passing through a typical interior point, $(x, y)$, in the flow field, and Eq. (2) spec-

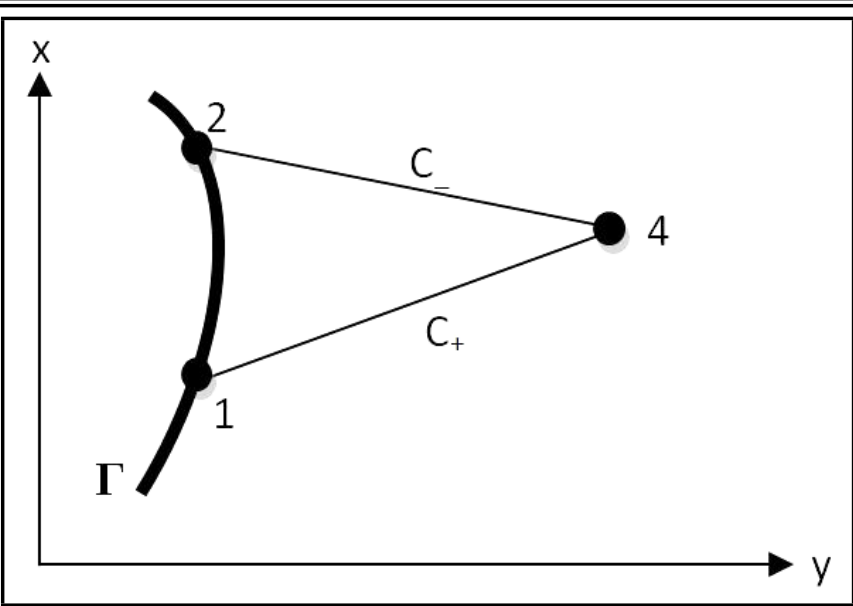

Figure 4. Solution at an interior point by the Method of Characteristics.

ifies one relationship between the velocity components $u$ and $v$ on each of the characteristics. In order to be able to find $u$ and $v$ at a given point, $(x, y)$, it is necessary to obtain two independent relationships between $u$ and $v$ at that point. This can be achieved by construction of a network such that two characteristics intersect at a common point. For example, consider the case shown in Fig. 4, where the velocity components $(u, v)$ are known at every point $(x, y)$ along the curve, $\Gamma$. The locations of points 1 and 2 are known, as are the velocity components there. The solution at a new point, 4 , is found by extending the $C_{+}$characteristic from point 1 and the $C_{-}$characteristic from point 2. The location of point $4,\left(x_{4}, y_{4}\right)$ and the solution there, $\left(u_{4}, v_{4}\right)$, are found by simultaneously solving the characteristic and compatibility equations, respectively.

From Eq. (3), the characteristic equations can be rewritten in terms of first-order finite difference equations as

$$
y_{4}-y_{1}=\lambda_{+}\left(x_{4}-x_{1}\right)
$$

and

$$
y_{4}-y_{2}=\lambda_{-}\left(x_{4}-x_{2}\right) .
$$

$\lambda_{+}$and $\lambda_{-}$are given by Eq. (3). Equations (4) and (5) can thus be solved for the two unknowns to yield the new location $\left(x_{4}, y_{4}\right)$. Recall that the compatibility equation, Eq. (2), is actually two equations. One is

$$
\begin{gathered}
\left(u_{+}^{2}-a_{+}^{2}\right) d u_{+}+\left[2 u_{+} v_{+}-\left(u_{+}^{2}-a_{+}^{2}\right) \lambda_{+}\right] d v_{+}- \\
\frac{a_{+}^{2} v_{+}}{y_{+}} d x_{+}=0
\end{gathered}
$$

which is valid along the $C_{+}$characteristic, (moving towards the flare surface) and the other comes from Fig. 4, which has known initial values

$$
u_{+}=u_{1}, \quad v_{+}=v_{1}, \quad y_{+}=y_{1} .
$$

Substituting Eq. (7) into Eq. (6), together with the first order finite difference approximation

$$
d x_{+}=x_{4}-x_{1}, \quad d u_{+}=u_{4}-u_{1}, \quad d v_{+}=v_{4}-v_{1}
$$

then Eq. (6) becomes a function of only two unknowns, $u_{4}$ and $v_{4}$. Similarly for the $C_{-}$characteristic (moving away from the flare surface). Solving these two equations yields predicted approximations to the location and velocity at a new point, 4 , 


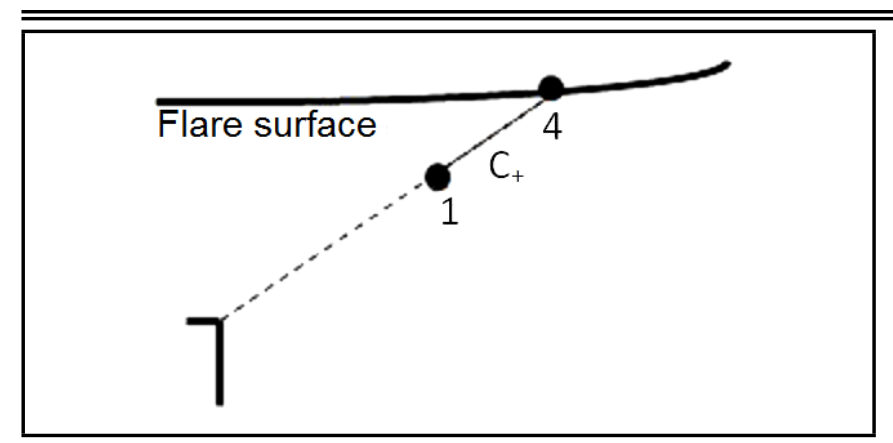

Figure 5. Notation used in MOC for wall points.

namely $x_{4}, y_{4}, u_{4}$ and $v_{4}$. These predictor values are then combined with the initial values to obtain average values of the flow properties using

$$
y_{+}=\frac{y_{1}+y_{4}}{2}, \quad u_{+}=\frac{u_{1}+u_{4}}{2}, \quad v_{+}=\frac{v_{1}+v_{4}}{2}
$$

and

$$
y_{-}=\frac{y_{2}+y_{4}}{2}, \quad u_{-}=\frac{u_{2}+u_{4}}{2}, \quad v_{-}=\frac{v_{2}+v_{4}}{2} .
$$

Note that $x$ is not needed, since it is absent in the compatibility equations. Average values of $\theta_{ \pm}, V_{ \pm}, a_{ \pm}, M_{ \pm}, \alpha_{ \pm}$and $\lambda_{ \pm}$ are then determined by substituting $u_{ \pm}$and $v_{ \pm}$from Eqs. (9) and (10) into the previous equations. Substitution of the values thus obtained into the characteristic Eqs. (4) and (5) yields the corrected values, $x_{4}$ and $y_{4}$. Insertion of these average values, together with the newly obtained corrected values $\left(x_{4}, y_{4}\right)$ and the first order finite difference approximations into the compatibility equations yields corrected values $u_{4}$ and $v_{4}$.

These correctors are then used with the initial values to recalculate the average values. Insertion of the new averages yields new corrector values. For a given point (e.g. point 4) the predictor-corrector method should be iterated in this way until the required degree of convergence is reached. This is typically when the difference between two successive sets of corrector values is below a pre-specified tolerance level. Once the flow characteristics for a given interior point are calculated, the entire process is repeated for the next point along the same characteristic in the direction from the nozzle lip to the flare surface $\left(C_{+}\right.$characteristics).

Once this entire characteristic is formed, the method returns to the nozzle lip and the next $C_{+}$characteristic (fanning away from the lip) and repeats the process. On completion of this fan of characteristics, the method locates the points where each of these characteristics meets the flare surface and, beginning with the left-most point, moves away from that point down a characteristic until it meets the jet boundary. The process is repeated for all these $C_{-}$characteristics until that fan is completed. The method then works from the ends of these $C_{-}$ characteristics (located at the jet boundary) back towards the flare surface, and continues in this way until the first shock wave forms.

\subsubsection{Wall Points}

In the case of the flare boundary, there are no $C_{-}$characteristics since they would lie inside the flare surface (see Fig. 5), and thus the characteristic equations are replaced by a single equation

$$
\lambda_{+}=\tan \left(\theta_{+}-\alpha_{+}\right) .
$$

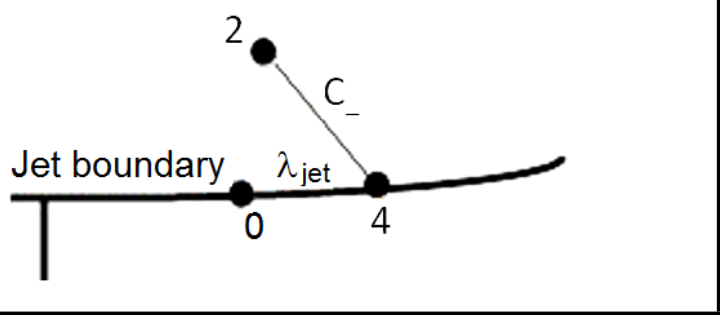

Figure 6. Notation used in MOC for jet boundary points.

Since $\theta_{+}$and $\alpha_{+}$are known, $\lambda_{+}$can be determined from Eq. (11).

The first order finite difference approximation given by Eq. (4) can be combined with the equation of the Coanda surface, for a given $R, d$ and $y_{0}$, namely

$$
y_{4}=\sqrt{R^{2}-\left(x_{4}-R-d\right)^{2}}+y_{0} ;
$$

and solved to obtain $x_{4}$ and $y_{4}$. Along the wall, interest is in $u_{+}$and $v_{+}$at the (now) known point $\left(x_{4}, y_{4}\right)$. Since $\left.\frac{d y}{d x}\right|_{\text {wall }}$ represents the slope of the Coanda surface at the known point $\left(x_{4}, y_{4}\right)$, then it is a known constant, $k$. Assuming that the flow has to remain attached to the wall- that is, the velocity follows the tangent to the wall- -then

$$
\frac{v_{4}}{u_{4}}=\left.\frac{d y}{d x}\right|_{\text {wall }}=k
$$

or

$$
v_{4}=k u_{4} \text {. }
$$

Substitution into the relevant compatibility equation yields the first approximation to the predictors $\left(x_{4}, y_{4}\right)$ and $\left(u_{4}, v_{4}\right)$. The predictor-corrector method is then iterated as described previously until the desired stopping criteria is reached.

\subsubsection{Jet Boundary Points}

For the jet boundary, there are no $C_{+}$characteristics (see Fig. 6), so we have only

$$
\lambda_{-}=\tan \left(\theta_{-}+\alpha_{-}\right) .
$$

Again, since $\theta_{-}$and $\alpha_{-}$are known, $\lambda_{-}$can be determined from Eq. (15). Now along the jet boundary

$$
\left.\frac{d y}{d x}\right|_{\text {jet }}=\lambda_{\text {jet }}=\frac{v_{0}}{u_{0}}
$$

where $\lambda_{\text {jet }}$ is approximated numerically by the (constant) slope of the secant line from the previous (known) point on the jet boundary, 0 , to the new point, 4 , namely

$$
\lambda_{\text {jet }}=\frac{y_{4}-y_{0}}{x_{4}-x_{0}}
$$

Thus Eq. (17) relates the two unknowns $x_{4}$ and $y_{4}$. Also recall from before that the first order finite difference approximation at the jet boundary is given by Eq. (5), which can be rewritten as

$$
\lambda_{-}=\frac{y_{4}-y_{2}}{x_{4}-x_{2}}
$$

Equations (17) and (18) are two equations with two unknowns $\left(x_{4}\right.$ and $\left.y_{4}\right)$ and can be solved to find $x_{4}$ and $y_{4}$. Work is currently underway to determine the jet boundary empirically as a 
function of slot width, $h$, and operating pressure, $p_{0}$. Once this is known, instead of using Eq. (17) to approximate $\lambda_{\text {jet }}$ numerically using the slope of the secant line between points 0 and 4 , the slope can be found analytically (as for the wall point).

Along a free pressure boundary, the total velocity is a function of the pressure, and since the pressure is atmospheric, the total velocity, $V_{\text {total }}$ is known. ${ }^{16}$ Thus

$$
u_{4}=\left(V_{\text {total }}^{2}-v_{4}^{2}\right)^{1 / 2}
$$

can be used together with the compatibility equation to determine $u_{4}$ and $v_{4}$. As previously, the predictor-corrector method is repeated until the desired stopping criteria is reached.

The method is initialized at the nozzle exit with the number of waves in the expansion fan, $N_{e}$, and the number of characteristics in the exit plane, $N_{c}$. Wherever they intersect, the location $(x, y)$ of these $N_{c}$ points and the velocity $(u, v)$ at each point is found. The method proceeds along the expansion line towards the flare surface (or jet boundary, depending on whether we are on a $C_{+}$or $C_{-}$characteristic) to find the next point. Once the flare surface is reached, the process is repeated for the next expansion line. As soon as the pre-specified number of lines in the expansion fan $\left(N_{e}\right)$ is reached, the method proceeds along their reflections from the flare to the jet boundary and repeats the process. Shock waves are formed where these lines coalesce. The method is based on the assumption that the flow in the expansion region is a simple wave. Figure 7 shows the shock cell structure predicted by the method described above under the operating conditions $p=60 \mathrm{psig}$, $U_{j x}=418 \mathrm{~m} / \mathrm{s}$, and $h=3.05 \mathrm{~mm}$.

Note the free-jet boundary displacement in Fig. 7. This agrees with the observed behaviour shown in Fig. 3 and described in Section 2. Work is currently underway to use experimental data to determine the equation of the flow boundary as a function of jet operating conditions. Input of this more accurate free-jet boundary (rather than the current assumption of a constant jet width, which is clearly highly unrealistic) will lead to an improved MOC model. Other methods of solving the MOC numerically are currently being investigated, and the associated results compared with those of the Euler predictorcorrector method.

\section{COMPARISON WITH EXPERIMENTAL RESULTS}

Experiments were conducted in a $5 \mathrm{~m} \times 2.5 \mathrm{~m} \times 2.5 \mathrm{~m}$ anechoic chamber. The Coanda surface had the following dimensions: $R_{c}=18.056 \mathrm{~mm}, R_{f}=9 \mathrm{~mm}$. The jet exit velocities $\left(U_{j x}\right)$ were between $200 \mathrm{~m} / \mathrm{s}$ and $500 \mathrm{~m} / \mathrm{s}$, and the exit slot (h) varied from $1.14 \mathrm{~mm}$ to $3.23 \mathrm{~mm}$. All experiments were carried out at ambient (room) temperature and pressure. Figure 3 shows the flow structure typically observed in the mixing layer region of the jet. Shock cells are clearly seen in the figure, and the interaction of large-scale coherent structures with these shock cells produces both broadband (BBSAN) and discretetone SAN, as shown in Fig. 8.

Figure 9 shows the predicted shock cell pattern superimposed on the previously described experimental results, for $U_{j x}=467 \mathrm{~m} / \mathrm{s}$, and $h=3.05 \mathrm{~mm}$. Other predictions exhibit similar features. The arrows represent the $(u, v)$ vectors at each point predicted by the intersections of characteristics,

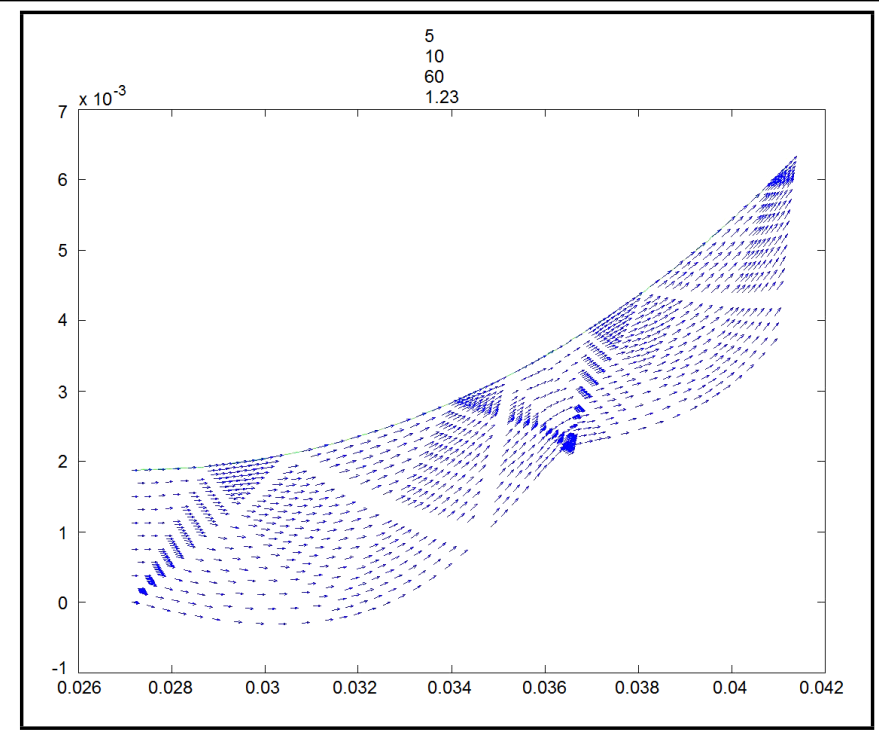

Figure 7. Predicted shock cell structure, $p=60 \mathrm{psig}, U_{j x}=418 \mathrm{~m} / \mathrm{s}$, $h=3.05 \mathrm{~mm}$.

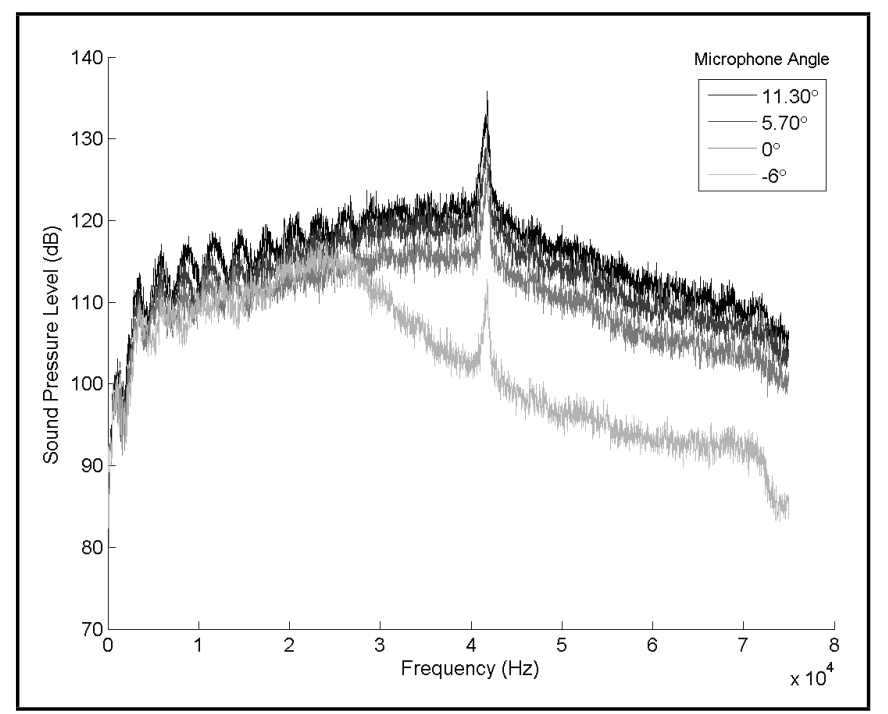

Figure 8. Flare Spectrum (54 mm diameter, $2.39 \mathrm{~mm}$ slot width, operating pressure 35 psig).

starting with the flow in the exit plane as well as the initial expansion fan. Shock waves are formed at the coalescence of these vectors, and both the dark regions on the flow visualization figures, and the higher concentration of vectors (shown in white) correspond to regions of higher pressure.

Comparison indicates that the preliminary model of SAN is relatively accurate at predicting the location of the first shock cell formation. ${ }^{24}$ Cells further from the exit slot are less well predicted, and future work will focus on modifying this preliminary model to include radial expansion and streamline curvature, which is anticipated to improve these predictions.

For reasons described previously, a key characteristic of interest is the location of the first shock cell and its dependence on flow characteristics. ${ }^{10,20,24}$ BBSAN has previously been shown to be independent of jet temperature. ${ }^{11}$ Figure 10 shows how the location of the first shock appears relatively unaffected by jet exit velocity, $U_{j x}$. Note that one of the reasons that it is extremely difficult to discern a clear relationship between shock cell behaviour and jet operating characteristics is the presence of occasional, but highly disruptive, discrete tones in 


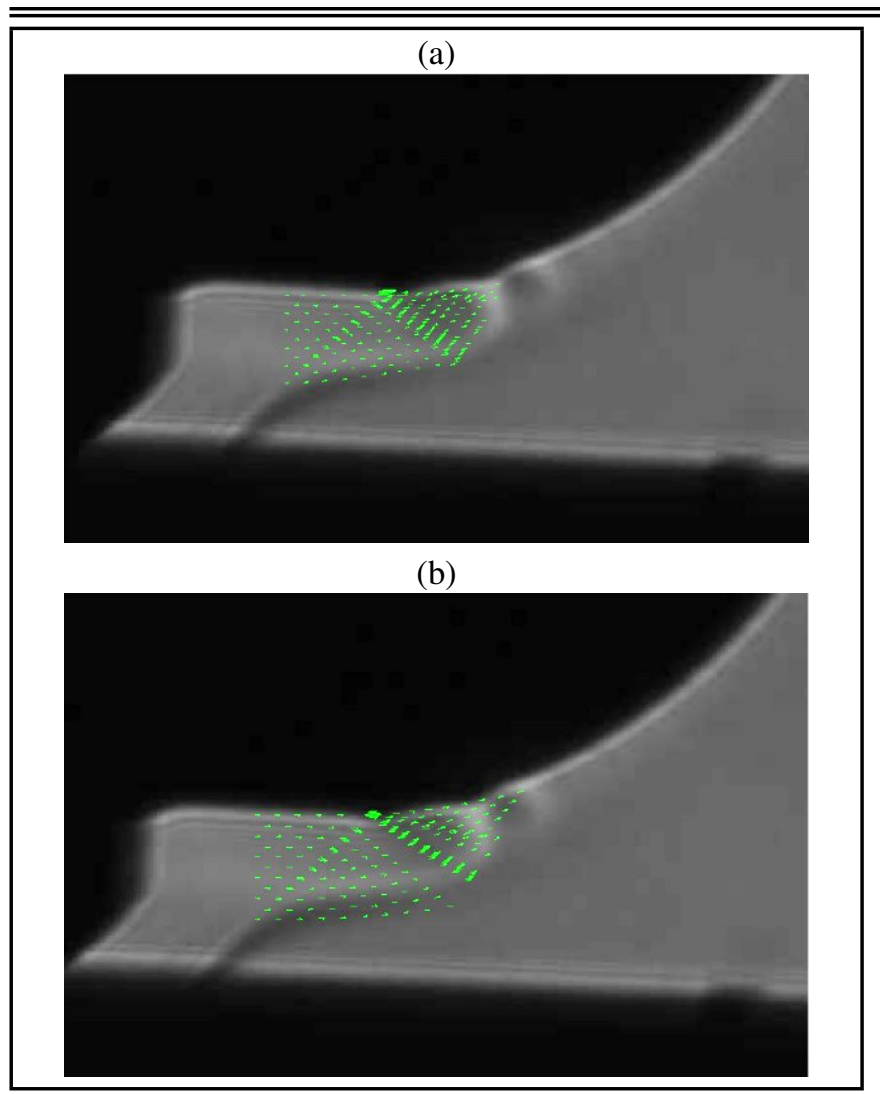

Figure 9. Comparison of experimental and theoretical results. (a) $h=$ $2.82 \mathrm{~mm} ; 30 \mathrm{psig}$ (b) $h=3.23 \mathrm{~mm} ; 35 \mathrm{psig}$.

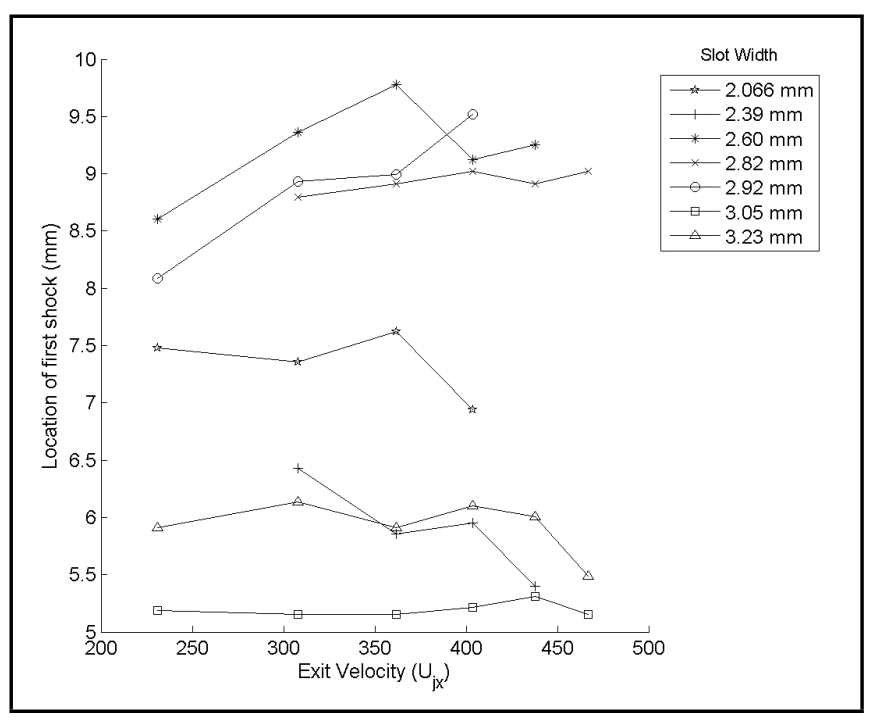

Figure 10. Variation in location of first shock cell with $U_{j x}$.

some of the experimental data. ${ }^{11,23-25}$ From Fig. 11 it appears that the location of the first shock cell is invariant with slot width. However, since the data are sparse, more experiments are needed to confirm this assertion, especially since Powell ${ }^{24}$ asserts that the location of the first shock cell is proportional to $h$.

As mentioned previously, typically 6-10 shock cells are formed in the exhaust of the turbulent Coanda flare jet, with shock cell spacing decreasing as we move away from the exit nozzle. Since the spacings do not vary too much, typically an average shock cell spacing, $L$, is used. Preliminary data on the variation of shock cell length from cell-to-cell (for the first

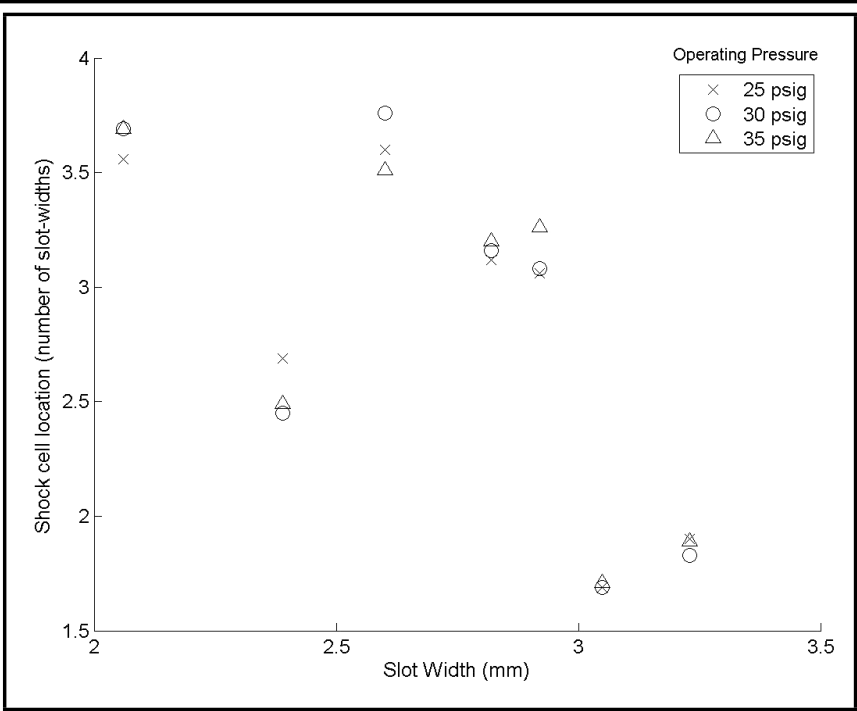

Figure 11. Variation in location of first shock cell with $h$.

five shocks) indicates that there is no clear pattern with respect to $U_{j x}$ or $p$ from shock to shock. Initial results indicate that the shock cell length is approximately equal to the slot width $h,{ }^{23}$ whereas Powell ${ }^{24}$ observed in the $2 \mathrm{D}$ case that $L$ is proportional to both $p$ and $h$. Harper-Bourne and Fisher ${ }^{11}$ suggest a possible relationship of the form

$$
L=1.1 \beta h
$$

where $\beta$ is the shock strength, which is a function of the local jet Mach number.

Breakaway is the phenomenon that is often observed in Coanda flows when a lip shock is formed at the lower edge of the exit slot. This lip shock generates a separation bubble on the Coanda surface that grows in size with operating pressure and ultimately causes the flow to separate from the Coanda surface to which it was formerly attached. ${ }^{7}$ When parameters such as operating pressure, nozzle exit slot width, and curvature are increased, the location of this separation bubble retreats around the Coanda surface back towards the nozzle exit, and, at some critical point, the flow breaks completely away from the surface. ${ }^{19}$ See Fig. 12 for details.

At high speeds, the separation process is usually further complicated by shock wave/boundary layer interaction. ${ }^{7}$ The breakaway process is shown in more detail by the set of Schlieren photos in Figs. 13(a)-13(d). In the present experimental work, for a given slot width, the operating pressure was continuously increased until breakaway was observed via the Schlieren flow visualisation system. The observed breakaway slot width-operating pressure (or exit velocity) combinations, are shown in Fig. 14. It should be noted that, for high speed Coanda flows, when the stagnation pressure is increased until breakaway occurs, and then decreased to make the flow reattach, it is found that the pressure has to be reduced to a considerably lower level than the breakaway pressure before reattachment occurs. This hysteresis effect occurs over a substantial range of operating pressures. ${ }^{7}$ Figure 15 shows how the SPL is affected by breakaway. As soon as breakaway occurs, a steep drop in SPL is observed, as the flow is redirected away from the horizontal. One of the motivations for the current interest in shock cell location (especially the location of the first shock cell, before the shock waves occur) is the potential for better 


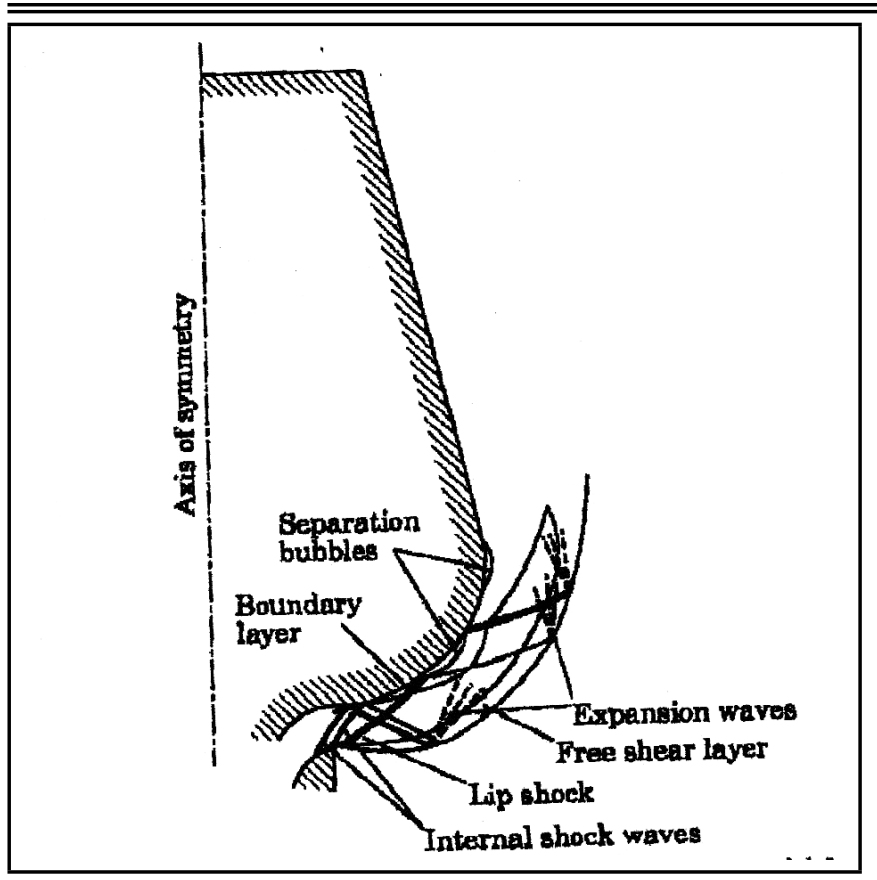

Figure 12. Schematic of key features of the flow field for a Coanda flow. ${ }^{7}$ (Reproduced with permission of reference [7]).

prediction of jet breakaway. ${ }^{20}$

Figure 16 shows the frequency spectra for $h=1.9 \mathrm{~mm}$ and a horizontal observer. For this slot width, breakaway occurs at $23 \mathrm{psig}$, and this is exhibited in Fig. 15 by the reduced SPL spectrum for $25 \mathrm{psig}$, compared to $20 \mathrm{psig}$. This is also exhibited in Fig. 16 by the lower SPL levels observed at $30 \mathrm{psig}$ and $40 \mathrm{psig}$ compared to $20 \mathrm{psig}$. The relationship between breakaway and location of the first shock cell is currently being investigated. As discussed previously, because of its complex nature, the flare jet is almost always imperfectly expanded. ${ }^{8}$ Indeed, the flow near the nozzle exit (especially for a stepped flare) is found to be supersonic and underexpanded at most operating pressures, ${ }^{10}$ leading to the formation of a series of shock cells. ${ }^{8}$ The interaction of the jet turbulence with these shock waves produces two noise components; screech (discrete) tones and BBSAN. ${ }^{11}$ Although BBSAN is generally present, the screech tones (which are thought to be produced by a feedback loop mechanism ${ }^{24}$ ) require sound waves of sufficient intensity to reach the jet nozzle exit region, or the loop cannot be sustained. Since such waves are not always present; ${ }^{8}$ the focus here is on BBSAN. Harper-Bourne and Fisher developed the first prediction method for BBSAN. ${ }^{11}$ In their model, the noise generation depends on a nearly coherent interaction between the turbulence in the jet shear layer and the jet shock cell structure. This interaction is modelled by a series of correlated point sources that radiate either constructively or destructively. It is well known that most of the SAN is produced in the region just downstream of the nozzle exit. ${ }^{10}$ Thus the location of the shock cells is of primary importance in determining both the BB shock cell noise spectrum and the peak frequency of the BBSAN emitted by a given jet configuration. ${ }^{11,22}$ For example, Harper-Bourne and Fisher ${ }^{11}$ have shown that the SAN peak frequency, $f_{p}$, is related to the (average) shock cell spacing, $L$, by

$$
f_{p}=\frac{U_{c}}{L\left(1-M_{c} \cos (\theta)\right)}
$$

where $U_{c}$ is the eddy convection velocity and $\left(1-M_{c} \cos (\theta)\right)$

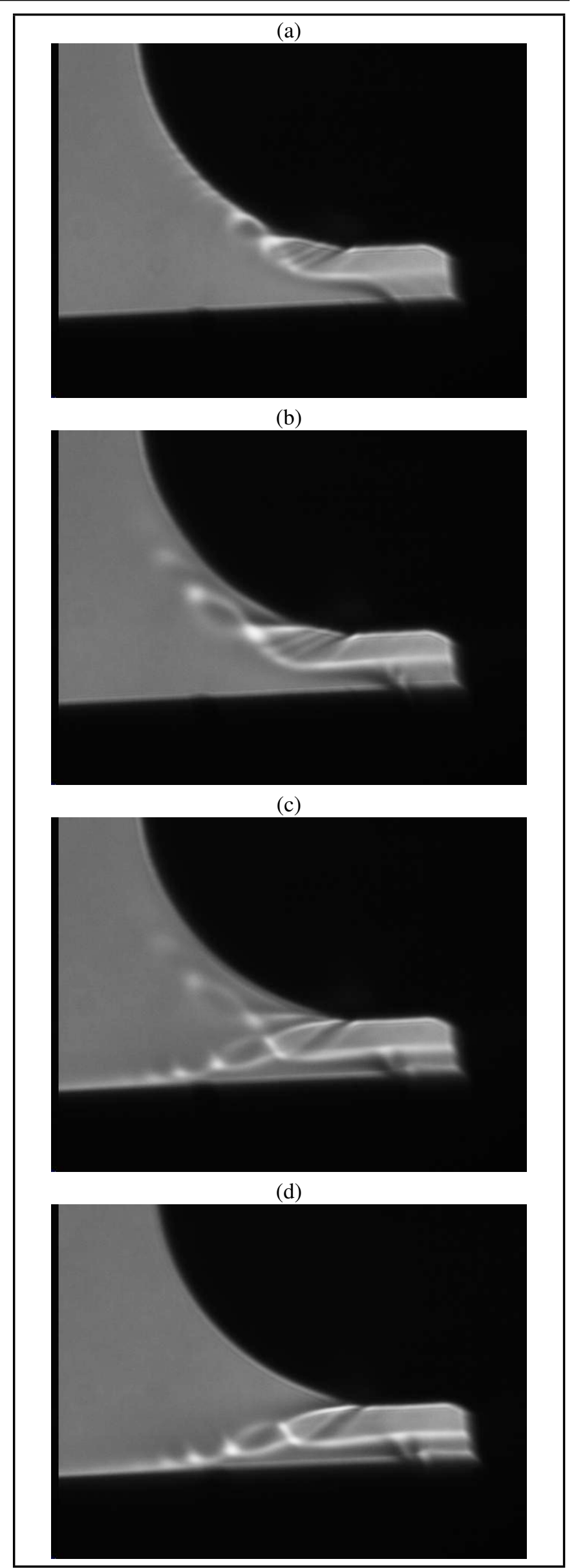

Figure 13. Schlieren photographs showing breakaway, $2.82 \mathrm{~mm}$ slot width (a) 45 psig (b) Before breakaway (c) During breakaway (d) After breakaway. 


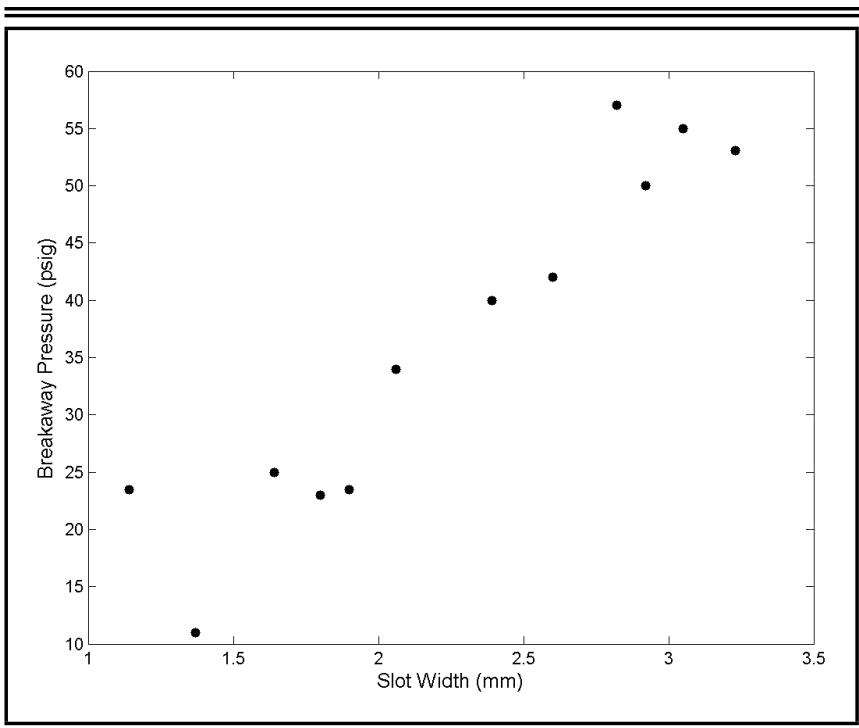

Figure 14. Variation in breakaway pressure with slot width.

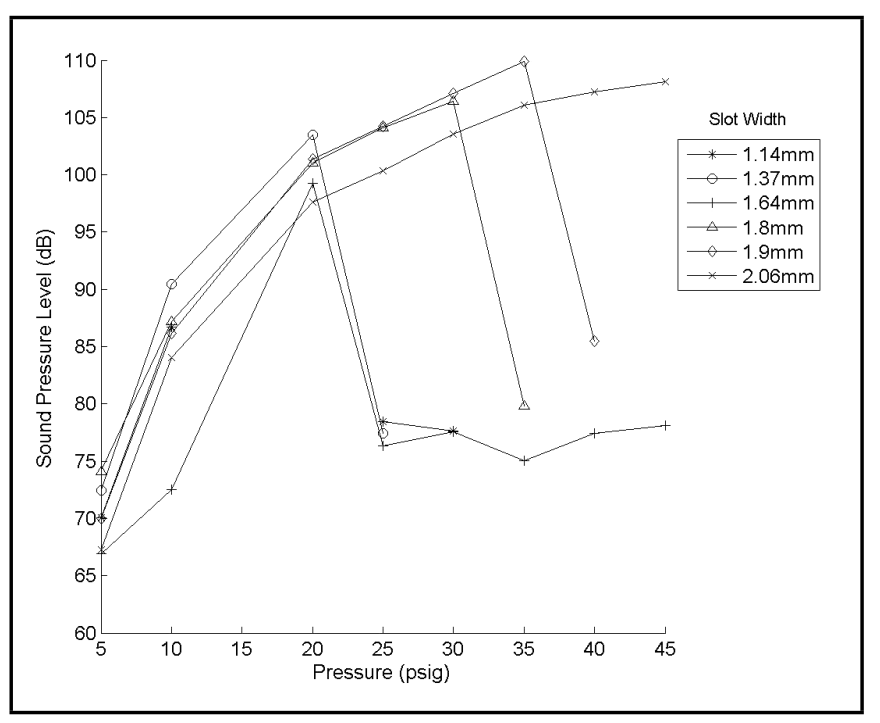

Figure 15. Effect of breakaway on SPL.

is a Doppler factor incorporating the variation in retarded time and source phasing. This agrees with the assertion of Pow$\mathrm{ell}^{24}$ for both a $2 \mathrm{D}$ nozzle and a round nozzle that the acoustic wavelength is inversely proportional to $L .{ }^{24}$

The current work is part of an effort to illuminate the relationship between cell location and flow characteristics such as operating pressure and slot width. Following the work of Tam et al., ${ }^{22}$ once a computational model has been developed to predict the shock cell structure in the jet, the Fourier modes of the shock cell structure can be calculated. Thus the interaction of the large scale turbulent structures with the shock cells (the source of BBSAN) can be thought of as the interaction of these structures with the different Fourier modes. Interaction with a particular mode generates a unique 'peak' in the shock cell noise spectrum, with the dominant peak usually generated by the first (fundamental) mode. ${ }^{22}$ The sound intensity and directionality can also be determined from the geometry of the sound field. ${ }^{24}$ Tam et al. ${ }^{22}$ obtained good agreement with experimental data by applying these methods to dual stream jets, although they note (as do the current authors) that clearly '... the computation of the shock cell structure is but the first step in the development of a shock cell

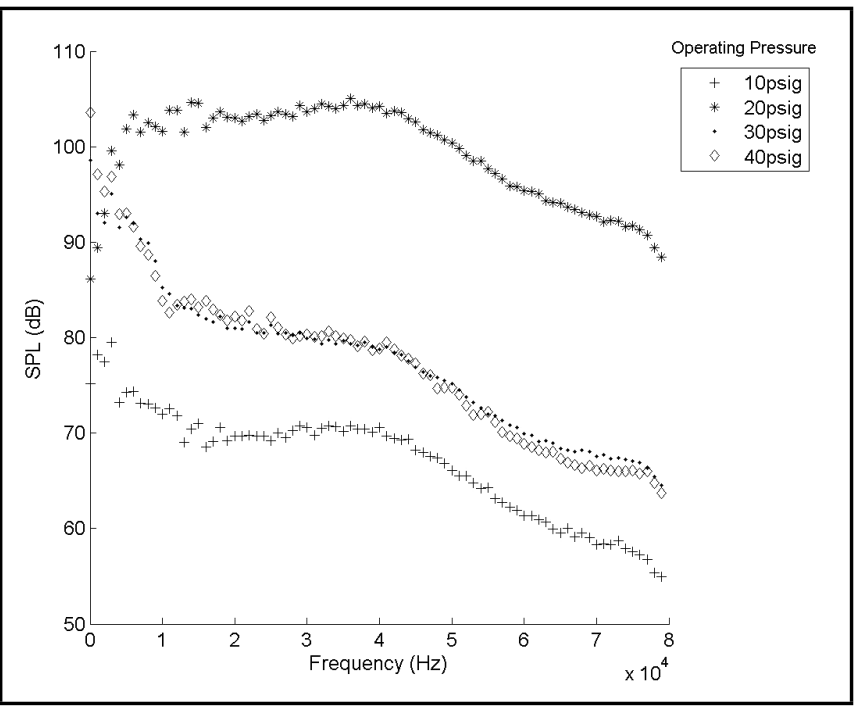

Figure 16. Effect of breakaway on frequency spectra, $1.9 \mathrm{~mm}$ slot width.

noise prediction methodology/theory'. Thus the current work is part of an ongoing research programme to combine the previous approaches ${ }^{11,20,24}$ to Coanda jets, and in this way (via the determination of the effect of jet operating conditions on shock cell structure), determine the effect of jet operating conditions on BBSAN. It should be noted that alternative models of BBSAN show reasonable agreement with the proposed approach. $^{12,13,22}$

\section{SUMMARY AND CONCLUSIONS}

A theoretical method of predicting the shock cell structure associated with a three-dimensional turbulent Coanda wall jet is presented, and compared with experimental data. The governing coupled quasi-linear partial differential equations are solved numerically using the MOC with the Euler predictorcorrector method. In addition to being a useful model in its own right, it has been noted ${ }^{10}$ that the MOC is useful in helping to interpret Schlieren photos. In this way, the location of the shock cells is determined. The qualitative displacement of the free-jet boundary is accurately modeled by the current method (Fig. 7), as are general features such as the relative invariance of shock cell location with $p$ and $h$ (Figs. 10 and 11). The relatively constant shock cell spacing is also observed in Fig. 7. The presence of discrete tones in the data disrupt observed trends, and means that the data are relatively sparse. Separation of the flow or breakaway is achieved across the entire range of operating conditions $(p=10-60$ psig, $h=1.14-3.23 \mathrm{~mm}$ ) at certain pressure/slot width combinations. It occurs at higher pressures for larger slot widths. The relationship between breakaway and the observed shock waves is currently being investigated in more detail by using the Schlieren images (see Fig. 13) in conjunction with the acoustical measurements. Sudden drops in SPL (see Fig. 15) denote that breakaway has occurred, and this is being correlated with the associated operating conditions in an attempt to determine whether such a relationship exists.

Clearly, the above work needs to be extended to better model three-dimensional turbulent Coanda wall jet flows. For example, three-dimensional jets require the inclusion of radial flow and longitudinal vortices. Indeed, such vortices have previously been utilised in Coanda flows to disrupt the coherent 
structures in a flow, thereby weakening the shock cell structure and reducing the OASPL. ${ }^{25}$ Curved $3 \mathrm{D}$ jets differ from plane 3D jets because they exhibit radial expansion and streamline curvature. Indeed, in a curved jet, the rapid growth of the outer shear layer (caused by streamline curvature) means that the shock cell structure is shorter than in equivalent plane jets. ${ }^{20}$ These features also tend to promote enhanced turbulence levels, and thus Coanda wall jet flows are even more complex than curved free jets, since they contain solid surface/shock wave and shock wave/boundary layer interactions. It is intended that future modelling work will incorporate many of these features.

\section{REFERENCES}

1 Lubert, C. P. On some recent applications of the Coanda effect, International Journal of Acoustics and Vibration, 16 (3), 144-153, (2011). http://dx.doi.org/10.20855/ijav.2011.16.3286

2 Coanda, H. Procédé de propulsion dans un fluide, Brevet d'Invention, Gr. 6 Cl. 2, no. 762688, République Française, (1932).

3 Reba, I. Applications of the Coanda effect, Scientific American, 214, 84-92, (1966). http://dx.doi.org/10.1038/scientificamerican0666-84

4 Lubert, C. P. Application of turbulent mixing noise theory to flows over Coanda surfaces, International Journal of Acoustics and Vibration, 13 (1), 17-30, (2008). http://dx.doi.org/10.20855/ijav.2008.13.1221

5 Desty, D. H. No smoke with fire, Proc. of the Institution of Mechanical Engineers, 197A, 159-170, (1983). http://dx.doi.org/10.1243/pime_proc_1983_197_018_02

6 Desty, D. H., Boden, J. C., and Witheridge, R. E. The origination, development and application of novel premixed flare burners employing the Coanda effect, $85^{\text {th }}$ National AIChE Meeting, Philadelphia, (1978).

7 Carpenter, P. W. and Green, P. N. The aeroacoustics and aerodynamics of high-speed Coanda devices, Part 1: Conventional arrangement of nozzle and surface, Journal of Sound and Vibration, 208 (5), 777-801, (1997). http://dx.doi.org/10.1006/jsvi.1997.1202

8 Parsons, C. An experimental and theoretical study of the aeroacoustics of external-Coanda gas flares, PhD Diss., University of Exeter, UK, (1988).

9 Smith, C. and Carpenter, P. W. The effect of solid surfaces on turbulent jet noise, Journal of Sound and Vibration, 185 (3), 397-413, (1995). http://dx.doi.org/10.1006/jsvi.1995.0388

10 Green, P. N. The fluid dynamics and aeroacoustics of external Coanda flares, PhD Diss., University of Exeter, UK, (1987)

11 Harper-Bourne, M. and Fisher, M. J. The noise from shockwaves in supersonic jets, AGARD Conference Proceedings, 131, Paper 11-1, (1973).
12 Tam, C. W. K. Stochastic model theory of broadband shock-associated noise from supersonic jets, Journal of Sound and Vibration, 116, 265-302, (1987). http://dx.doi.org/10.1016/s0022-460x(87)81303-2

13 Tam C. W. K. and Tanna, H. K. Shock-associated noise of supersonic jets from convergent-divergent nozzles, Journal of Sound and Vibration, 81 (3), 337-358, (1982). http://dx.doi.org/10.1016/0022-460x(82)90244-9

14 Morris, P. J. and Miller, S. A. E. The prediction of broadband shock-associated noise using RANS CFD, Proc. of the $15^{\text {th }}$ AIAA/CEAS Aeroacoustics Conference, Paper 20093315, (2009). http://dx.doi.org/10.2514/6.2009-3315

15 Miller, S. A. E. and Morris, P. J. The prediction of broadband shock-associated noise including propagation effects, International Journal of Aeroacoustics, 11 (7), (2011). http://dx.doi.org/10.2514/6.2011-2923

16 Zucrow, M. J. and Hoffman, J. D. Gas Dynamics; Volume II, Krieger, Florida, (1977).

17 Carpenter, P. W. and Green, P. N. The generation of noise in external Coanda-type waste-gas flares, Proc. of the Institute of Acoustics, 6 (1), (1984).

18 Bull, D. The shock associated noise of the Indair flare, Undergraduate Project Report, University of Exeter, UK, (1980).

19 Gilchrist, A. R. The development and breakaway of a compressible air jet with streamline curvature and its application to the Coanda flare, PhD Diss., Durham University, UK, (1985).

20 Gilchrist, A. R. and Gregory-Smith, D. G. Compressible Coanda wall jet: Predictions of jet structure and comparison with experiment, International Journal of Heat and Fluid Flow, 9 (3), 286-295, (1988). http://dx.doi.org/10.1016/0142-727x(88)90039-2

21 Dash, S. M., Beddini, R. A., Wolf, D. E., and Sinha, N. Viscous/inviscid analysis of curved sub- or supersonic wall jets, AIAA Journal, 23 (1), 12-13, (1985). http://dx.doi.org/10.2514/3.8865

22 Tam, C. K. W., Pastouchenko, N. N., and Viswanathan, $\mathrm{K}$. Computation of shock cell structure of dual-stream jets for noise prediction, AIAA Journal, 46 (11), (2008). http://dx.doi.org/10.2514/1.37045

23 Lubert, C. On some recent experimental results concerning turbulent Coanda wall jets, Proc. of Meetings on Acoustics, 22, (2015). http://dx.doi.org/10.1121/2.0000040

24 Powell, A. On the mechanism of choked jet noise, Proceedings of the Physical Society. Section B, 66, 1039-1056, (1953). http://dx.doi.org/10.1088/0370-1301/66/12/306

25 Carpenter, P. W. and Smith, C. The aeroacoustics and aerodynamics of high-speed Coanda devices, Part 2: Effects of modification for flow control and noise reduction, Journal of Sound and Vibration, 208 (5), 803-822, (1997). http://dx.doi.org/10.1006/jsvi.1997.1203 